\title{
Automated Management of Computer Management Procedure of Critical Control Point Information on Haccp System
}

\author{
Yongmei $\mathrm{Li}^{1}$, Yang Zhao ${ }^{2}$, Lingjun Wang ${ }^{1}$, Ying Wang ${ }^{* 1}$ \\ ${ }^{1}$ College of Economics and Management, Nanjing University of Aeronautics and Astronautics \\ Nanjing, 210016, China \\ ${ }^{2}$ Department of Electronic and Information Technology, Jiangmen Polytechnic \\ Jiangmen, 529090, China \\ \{wywy@ibhsedu.com\}
}

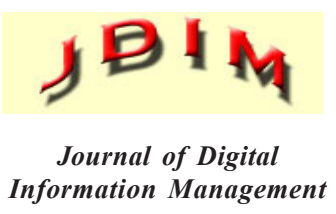

\begin{abstract}
Aiming to solve the management and decision-making problems occurring in food production field at present, this study strives to build an accurate and effective hazard analysis and critical control point (HACCP) computer automated management system combined with computer management programs by taking the HACCP system as standard. This system is able to be integrated into the overall framework of the system applying case-based reasoning (CBR) technology combined with ontological content and extended content, and emphasizes on exploring knowledge representation of HACCP system and computing method of similar cases, which improves the safety and order of food production.
\end{abstract}

Subject Categories and Descriptors

E.1 [Data Structures] Data Layer]; H.4 [Information Systems Applications] Service System

General Terms

Automated management, Service System

Keywords: Critical Control Point, Computer Management, HACCP

Received: 19 March 2020, Revised 4 July 2020, Accepted 19 July 2020

Review Metrics: Review Scale: 0/6, Review Score: 4.9, Inter-reviewer consistency: $78.2 \%$

DOI: $10.6025 / \mathrm{jdim} / 2021 / 19 / 1 / 27-30$

\section{Introduction}

With the rapid development of China's economy and the improvement of people's living standard, people put more and more attention to the food safety, so various domestic food manufacturers have begun to implement the HACCP safety system certification. HACCP, i.e., hazard analysis and critical control point, earliest proposed by the United Nations Food and Agriculture Organization (FAO) and the World Health Organization (WHO) in the end of last century, is responsible for evaluating risks in production process of food industry to make sure critical control point (CCP) - standard of food safety, thus taking certain means to eradicate the occurrence of food safety problems from the source [1].

Developed on the basis of brain thinking mode, casebased reasoning (CBR) technology solving problems by content or cases in on-hand inventory and similar computation is widely applied in food safety field [2]. CBR technology has supplementary and perfect effect on the HACCP system, improving the accuracy of the data, which makes up the deficiencies of complex process, large data size, etc. in the specific work of HACCP system [3].

As to above two deficiencies in HACCP system, this study sets up a HACCP knowledge service system based on ontology by applying advantages of ontological OWL language in knowledge formal description and sharing and CBR technology combined with traceability and HACCP system standard. It monitors and corrects error in the process of agricultural products production and processing, and also provides technical support for decision maker in semantic way to reduce the probability of harm, which is of significant practical meaning for further improving safety and traceability of agricultural products production.

\section{Structure Of HACCP Service System}




\subsection{Related introduction of HACCP system}

To establish HACCP system, seven steps needed are as follows: (1) CCP is confirmed as needed. (2) Hazard analysis is performed on problems that are likely to occur in confirmed CCP. (3) Critical limit related to CCP is confirmed. (4) Real-time monitoring is considered to be performed on specific operation event. (5) Corrective measures are taken on deviated event occurred in process. (6) Self validation procedure. (7) Program record and retention.

Other steps are to be carried out around CCP, core of establishing HACCP system. HACCP system is capable of reflecting the safety of food production data, but its uncontrollable working process plus coexistence of structural knowledge content and nonstructural knowledge of ontology make normal management of ontological knowledge system become a key point of establishing HACCP system.

\subsection{Framework of HACCP Service System}

Taking production data of dairy products processing enterprise as an example, this research builds knowledge service system of HACCP production quality and safety based on ontology (figure 1.1). All links in the entire system connect with one another, including application layer, logical relationship layer and data layer. Of them, application layer is divided into knowledge service, resource management and traceability system, etc., and data layer involving wide scope has connection with every link nearly. Resource management and traceability system refers to the application of relational database operation, and knowledge service is to manage knowledge structure in HACCP ontological base, and also effectively integrate and manage tree structure in order to help enterprise work out a HACCP plan and perform semantic analysis and information feedback.

Data layer mainly contains two parts of Structured Query

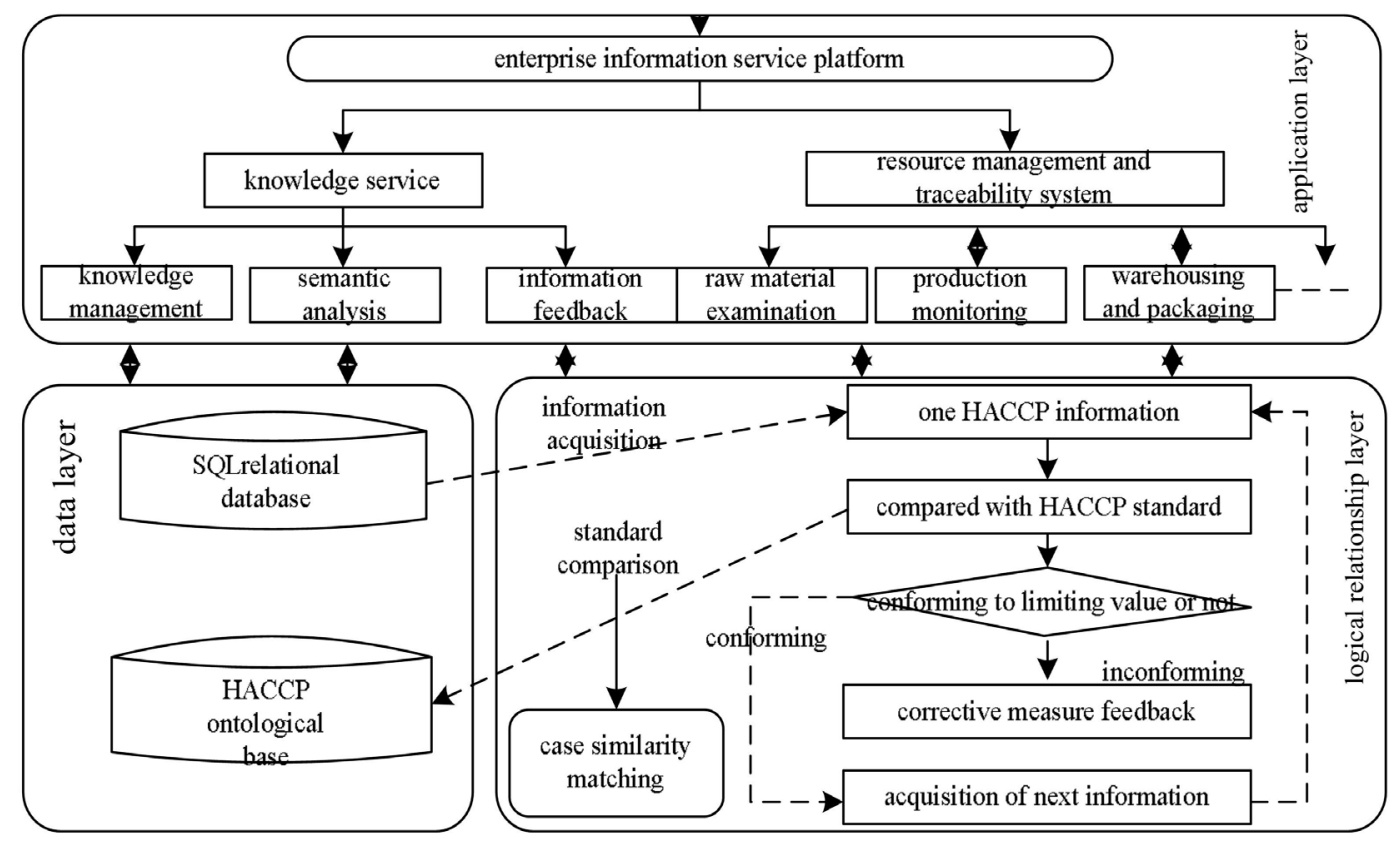

Figure 1. Framework of HACCP service system based on ontology and CBR

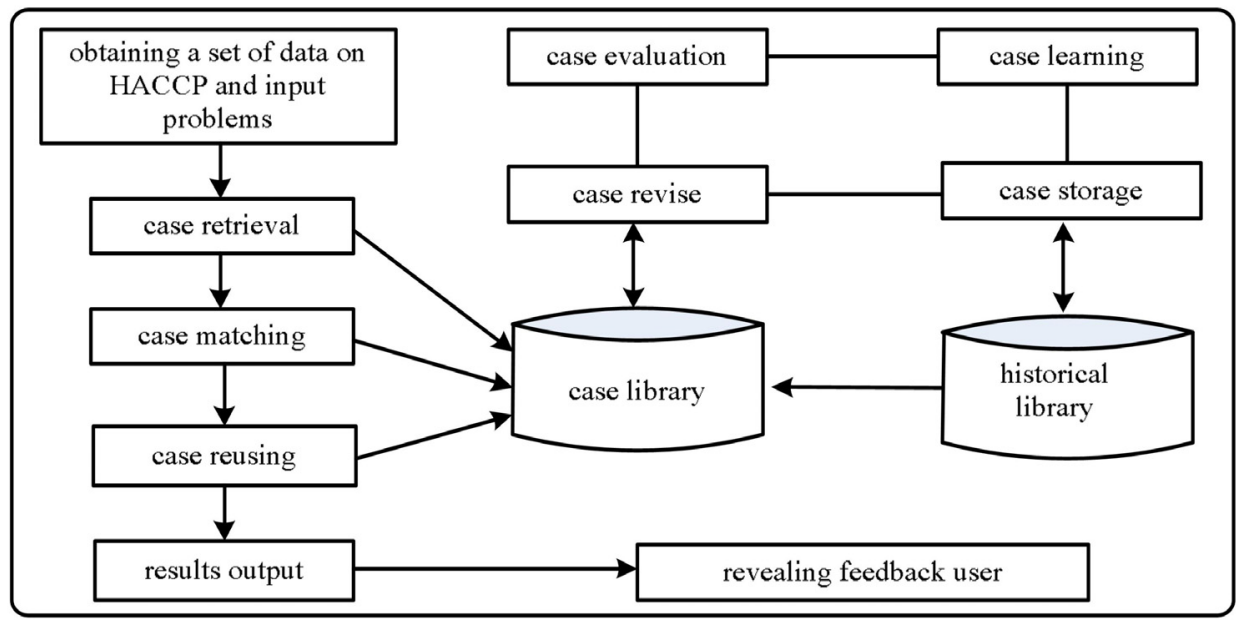

Figure 2. HACCP systems modeling of CBR technology 
Language (SQL) relational database and HACCP ontological base, in which, ontological base is a structural knowledge base expressed with ontological description language OWL including domain knowledge base and case knowledge base, while HACCP knowledge service is on the basis of existing data information in the relational database. On the foundation of working process and actual production processing operation process, logical relationship layer performs semantic similarity matching by obtaining existing data information and knowledge in HACCP knowledge base, and acquires solution of current problems through accessing to solution of the same case in knowledge, thus providing service support for decision maker.

\section{Technological Realization}

\subsection{HACCP System Modeling of CBR Technology}

Figure 2 shows the HACCP system modeling of CBR technology. HACCP system retrieves out the similar cases in case library according to the characters of problems after obtaining a piece of data, then performs similar screening and calculation. After calculation results are figured out, cases are evaluated, it is revised if the results are not conforming to system note, and otherwise it is saved.

\subsection{Expression and Organization of HACCP System}

Referring to literature [4], HACCP ontology is represented as a quintuple in definition 1, i.e., onto-HACCP: $=\{C, R$, $H, R e l, A\}$. In the formula, $\mathrm{C}$ (Concept) refers to concept set of domain forming collection $C s=\{C 1, C 2, C 3$, ......Cn $\}$. Additionally, concept set contains instance (I) forming instance set $I s=\{I 1, I 2, I 3, \ldots \ldots\}$, in the meantime, includes $\mathrm{n}$ subsets of Cartesian product, $R: C 1 \times$ $\mathrm{C} 2 \times \ldots \times C n$.

In OWL, attribute relationship is expressed with rdf of RDF: subclass of Property. $\mathrm{H}$ is on behalf of the taxonomy relation of different types, and the whole system is able to be divided into well-bedded reticular structure according to different functions. Rel stands for differences of various concepts. A (Axiom) representing collection of ontology is the foundation of system calculation, and applied in identifying the case similarity and choosing the appropriate calculation method.

As to definition 2, the ontology contains numerous cases in HACCP system. This research expresses case as a tetrad drawing a lesson from frame knowledge representation put forward by Minsky [5]: CCP, critical limit, hazard analysis and corrective measure.

\subsection{Case Similarity Calculation based on Ontology}

To realize the accuracy of assessment data and correctly screen suitable case calculation method, a lot of researches on ontological knowledge system matching are done when evaluating food safety, mainly in the perspective of the overall and individual case relationship of the food safety production.
(1) Calculation of weighted average of overall food safety production

$$
A \operatorname{sim}=\sum_{i=1}^{n} \operatorname{sim}\left(x_{i}, y_{i}\right) \times w(i) / \sum_{i=1}^{n} w(i)
$$

Of them, $n$ is the number of each attribute, simxiyi is on behalf of the similarity of the ith simple attribute in cases, $w_{(i)}$ expresses the weight of the ith attribute.

(2) Calculation of vector similarity of individual food safety production

One case character expressed as a data structure is divided into multiple characteristic components, defined as $x=\left(x_{1}, x_{2} \ldots x_{n}\right)$, in which, $n$ is dimension of vector.

When the characteristic value is a value type, assuming there are two cases of $x$ and $y, x=\left(x_{1}, x_{2} \ldots \ldots x_{n}\right)$ refers to newly obtained case in relational data base, and $y=\left(y_{1}\right.$, $\left.y_{2} \ldots \ldots . y_{n}\right)$ is the existing case in case ontology base, the calculation formula is:

When the characteristic value is a value type, two new cases $x i, y i$ depend on $[a, b]$, then the similarity of two cases is:

$$
\operatorname{sim}(x, y)=1-\frac{\left|x_{i}-y_{i}\right|}{a-b}
$$

When data occurs in the form of character, complex processing without response to character string calculation is only based on keyword because the system performs data matching by similarity [6]. Assume vector space made up of effective keywords of two cases $(x, y)$ is $v=\left(k_{1}, k_{2} \ldots \ldots . k_{n}\right)$, and $k i$ is the effective keyword. However, vector quantity of $x$ and $y$ is $v_{1}=\left(a_{1}, a_{2} \ldots \ldots \ldots a_{n}\right), v_{2}=$ $\left(b_{1}, b_{2} \ldots . . b_{n}\right)$ respectively, $a i$ and $b i$ are effective keywords orderly, and ki refers to the frequency of occurrence in two character strings. Therefore, the similarity of $x$ and $y$ is as follows after saving case whose similarity value is over 0.4 into candidate set:

$$
\operatorname{sim}(x, y)=\frac{\sum_{i=1}^{n} a_{i} \times b_{i}}{\sqrt{\left(\sum_{i=1}^{n} a_{i}^{2}\right)\left(\sum_{i=1}^{n} b_{i}^{2}\right)}}
$$

When selected data is a concept, ontology itself contains this content. Each point location in concept tree is able to be matched with data, and look for suitable similarity with following calculation formula [6]:

$$
\operatorname{sim}(x, y)=\frac{\max (\operatorname{Deep}(\operatorname{Lcs}(x, y)))}{\max \left(\operatorname{Deep}\left(C_{i}\right)\right), C_{i} \in C_{n}}
$$

Of them, DeepCi stands for the depth of concept or instance in the tree, $\operatorname{Lcs}(x, y)$ explains the nearest parent concept to concept or instance. 


\begin{tabular}{|l|l|l|l|}
\hline CCP & Critical limit & Hazard analysis & Corrective measure \\
\hline $\begin{array}{l}\text { Raw material } \\
\text { inspection }\end{array}$ & $\begin{array}{l}\text { HNFF, color and lustre, } \\
\text { enzyme value... }\end{array}$ & $\begin{array}{l}\text { Physical hazard, chemical haz- } \\
\text { ard, biological hazard }\end{array}$ & $\begin{array}{l}\text { Rejecting raw material offered by sup- } \\
\text { pliers without test report, rejecting } \\
\text { products without organic authentication }\end{array}$ \\
\hline $\begin{array}{l}\text { Production } \\
\text { monitoring }\end{array}$ & $\begin{array}{l}\text { Temperature, filtration, } \\
\text { vacuum degree... }\end{array}$ & $\begin{array}{l}\text { With or without ponding on the } \\
\text { ground, with or without insects } \\
\text { flying into workshop }\end{array}$ & $\begin{array}{l}\text { Changing filter net immediately when } \\
\text { critical limit deviates from specified } \\
\text { requirements, reworking products miss } \\
\text { critical limit. }\end{array}$ \\
\hline
\end{tabular}

Table 1. Information sheet of hazard measures and corrective measures

\section{Application Instance}

A prototype system of HACCP knowledge service system based on ontology is realized by taking protégé [7] as ontological editing tool and applying Eclipse and JenaAPI software developer's kit of java technical platform. On the foundation of bee product processing enterprise resource management (ERM) and traceable data, it is divided into three modules of raw material, production and finished product, and also contains newly increased and modification instance module for decision maker making HACCP plan.

From table 1, we can figure out that this study matches case with high similarity after confirming CCP, then compares the relationship between instance and case. Here, testing options of batch number from 10001 to 10005 are selected, in which, moisture is 6.0 , and color and lustre is 6 , standard limiting value of moisture test item in the ontological knowledge base is 0.20 , and standard limiting value of color and lustre is 5.0. Similar case information is looked for in ontology, and finally corresponding information is worked out after calculation.

Lists hazard analysis and corrective measure results of two CCPs in raw material inspection and production monitoring process in actual operation, verifying the effectiveness and practicability of knowledge service.

\section{Conclusion}

This research builds a HACCP knowledge service system based on ontology, performs knowledge representation for knowledge and process of each step in HACCP system, and describes their structural relationship precisely, also sets up onto-HACCP and realizes prototype system verification. Traceability information is fully combined with HACCP standard system, and both of them are applied in bee product processing ERM and trace ability system, which offers knowledge service to some extent, and achieves real-time monitoring and decisionmaking purpose. But, due to their inaccurate semantic similarity matching and excessive similar case scope fed back by system, more detailed researches in fuzzy reasoning are about to be done and standards, such as GAP, ISO2000, etc. are going to be introduced in order to perform knowledge description and knowledge service.

\section{References}

[1] Yanlan, Su., Gongde, Liu. (2013). HACCP Application in Tropical Fruits Pellet. J. Popular Science \& Technology. (8) 58-60.

[2] Jinyong, Sun., Tianlong, Gu., Liang, Chang. (2014). Survey of Case-based Reasoning Based on Description Logics. J. Computer Science. 41 (11) 1-6, 39.

[3] Ying, Zhang. (2014). The Effect of HACCP System on Sterilized Milk Production. J. China Science and Technology Review. (2) 350-350.

[4] Jiamin, Yao., Sichun, Yang. (2013). Improved Model for Similarity Computation of Ontology Concept. J. Journal of Computer Applications. 33 (6) 1579-1581. 2013.

[5] Huimin, Fan., Shangbing, Wang., Zhen, Huo. (2010). Domain Knowledge Representation of Boiler Fault Diagnosis Expert System. J. Computer Knowledge and Technology (Academic Exchange). 06 (8) 6292-6294. 2010.

[6] Ying, Zhang., Xin, Li. (2013). Keyword Search and Results Ranking over Relational Databases. J. Journal of Liaoning Institute of Technology (Natural Science Edition). 33 (5) 305-309. 2013.

[7] Sherry, N., Hagopian, W., Ludvigsson, J. (2011). Teplizumab for Treatment of Type 1 Diabetes (Protégé Study): One-year Results from a Randomised, Placebocontrolled Trial. J. The Lancet. 378 (9790): 487-497. 